International Journal of Pure and Applied Mathematics

Volume 106 No. 2 2016, 583-591

ISSN: 1311-8080 (printed version); ISSN: 1314-3395 (on-line version)

url: http://www.ijpam.eu

doi: 10.12732/ijpam.v106i2.21

\title{
A NOTE ON THE GUTMAN INDEX OF JACO GRAPHS
}

\author{
Johan Kok ${ }^{1 \S}$, C. Susanth ${ }^{2}$, Sunny Joseph Kalayathankal ${ }^{3}$ \\ ${ }^{1}$ Tshwane Metropolitan Police Department \\ City of Tshwane, REPUBLIC OF SOUTH AFRICA \\ ${ }^{2}$ Department of Mathematics \\ Vidya Academy of Science and Technology \\ Thalakkottukara, Thrissur-680501, INDIA \\ ${ }^{3}$ Department of Mathematics \\ Kuriakose Elias College \\ Mannaman, Kottayam-686561, INDIA
}

\begin{abstract}
The concept of the Gutman index, denoted Gut $(G)$ was introduced for a connected undirected graph $G$. In this note we apply the concept to the underlying graphs of the family of Jaco graphs, (directed graphs by definition), and decribe a recursive formula for the Gutman index $G u t\left(J_{n+1}^{*}(x)\right)$. We also determine the Gutman index for the trivial edge-joint between Jaco graphs.
\end{abstract}

AMS Subject Classification: 05C12, 05C20, 05C38, 05C40, 05C75

Key Words: Gutman index, Jaco graph, edge-joint

\section{Introduction}

For general reference to notation and concepts of graph theory see [2]. Unless mentioned otherwise, a graph $G=G(V, E)$ on $\nu(G)$ vertices (order of $G$ ) with $\epsilon(G)$ edges (size of $G$ ) will be a finite undirected and connected simple graph. The degree of a vertex in $G$ is denoted $d_{G}(v)$ and if the context of $G$ is clear the degree is denoted $d(v)$ for brevity. Also in a directed graph $G^{\rightarrow}$ the degree is $d_{G \rightarrow}(v)=d_{G \rightarrow}^{+}(v)+d_{G \rightarrow}^{-}(v)$ or for brevity, $d(v)=d^{+}(v)+d^{-}(v)$ if $G$ is clear.

Received: November 25, 2015

Published: February 15, 2016

$\S$ Correspondence author (c) 2016 Academic Publications, Ltd.

url: www.acadpubl.eu 
The concept of the Gutman index $G u t(G)$ of a connected undirected graph $G$ was introduced in 1994 by Gutman [4]. It is defined to be $\operatorname{Gut}(G)=$ $\sum_{\{v, u\} \subseteq V(G)} d_{G}(v) d_{G}(u) d_{G}(v, u)$, where $d_{G}(v)$ and $d_{G}(u)$ are the degree of $v$ and $u$ in $G$ respectively, and $d_{G}(v, u)$ is the distance between $v$ and $u$ in $G$. Clearly, if the vertices of $G$ of order $n$ are randomly labeled $v_{1}, v_{2}, v_{3}, \ldots, v_{n}$ the definition states that $G u t(G)=\sum_{\ell=1}^{n-1} \sum_{j=\ell+1}^{n} d_{G}\left(v_{\ell}\right) d_{G}\left(v_{j}\right) d_{G}\left(v_{\ell}, v_{j}\right)$. Worthy results are reported in Andova et al. [1] and Dankelmann et al. [3].

\section{The Gutman Index of the Underlying Graph of a Jaco Graph}

Despite earlier definitions in respect of the family of Jaco graphs $[5,6]$, the definitions found in [7] serve as the unifying definitions. For ease of reference some of the important definitions are repeated here.

Definition 2.1. [7] Let $f(x)=m x+c ; x \in \mathbb{N}, m, c \in \mathbb{N}_{0}$. The family of infinite linear Jaco graphs denoted by $\left\{J_{\infty}(f(x)): f(x)=m x+c ; x \in \mathbb{N}\right.$ and $\left.m, c \in \mathbb{N}_{0}\right\}$ is defined by $V\left(J_{\infty}(f(x))\right)=\left\{v_{i}: i \in \mathbb{N}\right\}, A\left(J_{\infty}(f(x))\right) \subseteq\left\{\left(v_{i}, v_{j}\right)\right.$ : $i, j \in \mathbb{N}, i<j\}$ and $\left(v_{i}, v_{j}\right) \in A\left(J_{\infty}(f(x))\right)$ if and only if $(f(i)+i)-d^{-}\left(v_{i}\right) \geq j$.

Definition 2.2. [7] The family of finite linear Jaco graphs denoted by $\left\{J_{n}(f(x)): f(x)=m x+c ; x \in \mathbb{N}\right.$ and $\left.m, c \in \mathbb{N}_{0}\right\}$ is defined by $V\left(J_{n}(f(x))\right)=$ $\left\{v_{i}: i \in \mathbb{N}, i \leq n\right\}, A\left(J_{n}(f(x))\right) \subseteq\left\{\left(v_{i}, v_{j}\right): i, j \in \mathbb{N}, i<j \leq n\right\}$ and $\left(v_{i}, v_{j}\right) \in$ $A\left(J_{n}(f(x))\right)$ if and only if $(f(i)+i)-d^{-}\left(v_{i}\right) \geq j$.

The reader is referred to [7] for the definition of the prime Jaconian vertex and the Hope graph. The graph has four fundamental properties which are:

(i) $V\left(J_{\infty}(f(x))\right)=\left\{v_{i}: i \in \mathbb{N}\right\}$ and,

(ii) if $v_{j}$ is the head of an arc then the tail is always a vertex $v_{i}, i<j$ and,

(iii) if $v_{k}$, for smallest $k \in \mathbb{N}$ is a tail vertex then all vertices $v_{\ell}, k<\ell<j$ are tails of arcs to $v_{j}$ and finally,

(iv) the degree of vertex $k$ is $d\left(v_{k}\right)=f(k)$.

The family of finite directed graphs are those limited to $n \in \mathbb{N}$ vertices by lobbing off all vertices (and arcs to vertices) $v_{t}, t>n$. Hence, trivially $d\left(v_{i}\right) \leq i$ for $i \in \mathbb{N}$. For $m=0$ and $c \geq 0$ two special classes of disconnected linear Jaco graphs exist. For $c=0$ the Jaco graph $J_{n}(0)$ is a null graph (edgeless graph) on $n$ vertices. For $c>0$, the Jaco graph $J_{n}(c)=\underset{\left\lfloor\frac{n}{c+1}\right\rfloor-\text { copies }}{\bigcup} K_{c+1} \bigcup K_{n-(c+1) \cdot\left\lfloor\frac{n}{c+1}\right\rfloor}$. 
since the Gutman index is defined for connected graphs the bound $m \geq 1$ will apply.

In this note we only consider the case $m=1, c=0$. The generalisation for $f(x)=m x+c$ in general remains open. Denote the underlying Jaco graph by $J_{n}^{*}(f(x))$. A recursive formula of the Gutman index $G u t\left(J_{n+1}^{*}(x)\right)$ in terms of $\operatorname{Gut}\left(J_{n}^{*}(x)\right)$ is given in the next theorem.

Theorem 2.1. For the underlying graph $J_{n}^{*}(x)$ of a finitie Jaco Graph $J_{n}(x), n \in \mathbb{N}, n \geq 2$ with Jaconian vertex $v_{i}$ we have that recursively:

$$
\begin{aligned}
& \operatorname{Gut}\left(J_{n+1}^{*}(x)\right)=\operatorname{Gut}\left(J_{n}^{*}(x)\right)+\sum_{k=1}^{i} \sum_{t=i+1}^{n} d_{J_{n}^{*}(x)}\left(v_{k}\right) d_{J_{n}^{*}(x)}\left(v_{k}, v_{t}\right) \\
& +\sum_{t=i+1}^{n-1} \sum_{q=t+1}^{n}\left(d_{J_{n}^{*}(x)}\left(v_{t}\right)+d_{J_{n}^{*}(x)}\left(v_{q}\right)\right)+(n-i)\left(\sum_{k=1}^{i} d_{J_{n}^{*}(x)}\left(v_{k}\right) d_{J_{n}^{*}(x)}\left(v_{k}, v_{n}\right)\right. \\
& \left.+\sum_{t=i+1}^{n} d_{J_{n}^{*}(x)}\left(v_{t}\right)\right)+(n-i-1)+i(n-i) .
\end{aligned}
$$

Proof. Consider the underlying Jaco graph, $J_{n}^{*}(x), n \in \mathbb{N}, n \geq 2$ with prime Jaconian vertex $v_{i}$. Now consider $J_{n+1}^{*}(x)$. From the definition of a Jaco graph the extension from $J_{n}^{*}(x)$ to $J_{n+1}^{*}(x)$ adds the vertex $v_{n+1}$ and the edges $v_{i+1} v_{n+1}, v_{i+2} v_{n+1}, \ldots, v_{n} v_{n+1}$.

Step 1: Consider any ordered pair of vertices $\left(v_{k}, v_{q}\right)_{k<q}, 1 \leq k \leq i-1$, and $k+1 \leq q \leq i$. By applying the definition of the Gutman index to this pair of vertices we have the term:

$$
d_{J_{n+1}^{*}(x)}\left(v_{k}\right) d_{J_{n+1}^{*}(1)}\left(v_{q}\right) d_{J_{n+1}^{*}(x)}\left(v_{k}, v_{q}\right)=d_{J_{n}^{*}(x)}\left(v_{k}\right) d_{J_{n}^{*}(x)}\left(v_{q}\right) d_{J_{n}^{*}(x)}\left(v_{k}, v_{q}\right) .
$$

By applying this step $\forall v_{k}, 1 \leq k \leq i-1$, and $\forall v_{q}, k+1 \leq q \leq i$ with $k<q$ we obtain:

$$
\sum_{k=1}^{i-1} \sum_{q=k+1}^{i} d_{J_{n}^{*}(x)}\left(v_{k}\right) d_{J_{n}^{*}(x)}\left(v_{q}\right) d_{J_{n}^{*}(x)}\left(v_{k}, v_{q}\right) .
$$

Step 2: Consider any vertex $v_{k}, 1 \leq k \leq i$ and any other vertex $v_{t}, i+1 \leq$ $t \leq n$. By applying the definition of the Gutman index to this pair of vertices we have the term:

$$
\begin{array}{r}
d_{J_{n+1}^{*}(x)}\left(v_{k}\right) d_{J_{n+1}^{*}(x)}\left(v_{t}\right) d_{J_{n+1}^{*}(x)}\left(v_{k}, v_{t}\right)=d_{J_{n}^{*}(x)}\left(v_{k}\right)\left(d_{J_{n}^{*}(x)}\left(v_{t}\right)+1\right) d_{J_{n}^{*}(x)}\left(v_{k}, v_{t}\right) \\
=d_{J_{n}^{*}(x)}\left(v_{k}\right) d_{J_{n}^{*}(x)}\left(v_{t}\right) d_{J_{n}^{*}(x)}\left(v_{k}, v_{t}\right)+d_{J_{n}^{*}(x)}\left(v_{k}\right) d_{J_{n}^{*}(x)}\left(v_{k}, v_{t}\right)
\end{array}
$$


By applying this step $\forall v_{k}, 1 \leq k \leq i$ and $\forall v_{t}, i+1 \leq t \leq n$, we obtain:

$$
\sum_{k=1}^{i} \sum_{t=i+1}^{n} d_{J_{n}^{*}(x)}\left(v_{k}\right) d_{J_{n}^{*}(x)}\left(v_{t}\right) d_{J_{n}^{*}(x)}\left(v_{k}, v_{t}\right)+\sum_{k=1}^{i} \sum_{t=i+1}^{n} d_{J_{n}^{*}(x)}\left(v_{k}\right) d_{J_{n}^{*}(x)}\left(v_{k}, v_{t}\right) .
$$

Step 3: Consider any two distinct vertices $v_{t}, v_{q}, i+1 \leq t \leq n-1$, and $t+1 \leq q \leq n$. By applying the definition of the Gutman index to this pair of vertices we have the term:

$$
\begin{aligned}
d_{J_{n+1}^{*}(x)}\left(v_{t}\right) d_{J_{n+1}^{*}(x)}\left(v_{q}\right) & d_{J_{n+1}^{*}(x)}\left(v_{t}, v_{q}\right) \\
=\left(d_{J_{n}^{*}(x)}\right. & \left.\left(v_{t}\right)+1\right)\left(d_{J_{n}^{*}(x)}\left(v_{q}\right)+1\right) d_{J_{n}^{*}(x)}\left(v_{t}, v_{q}\right) \\
& =d_{J_{n}^{*}(x)}\left(v_{t}\right) d_{J_{n}^{*}(x)}\left(v_{q}\right)+d_{J_{n}^{*}(x)}\left(v_{t}\right)+d_{J_{n}^{*}(x)}\left(v_{q}\right)+1 .
\end{aligned}
$$

By applying this step $\forall v_{t}, i+1 \leq t \leq n-1$ and $\forall v_{q}, t+1 \leq q \leq n$, we obtain:

$$
\begin{aligned}
& \sum_{t=i+1}^{n-1} \sum_{q=t+1}^{n} d_{J_{n}^{*}(x)}\left(v_{t}\right) d_{J_{n}^{*}(x)}\left(v_{q}\right) \\
& \quad+\sum_{t=i+1}^{n-1} \sum_{q=t+1}^{n}\left(d_{J_{n}^{*}(x)}\left(v_{t}\right)+d_{J_{n}^{*}(x)}\left(v_{q}\right)\right)+(n-i-1) .
\end{aligned}
$$

Step 4: Consider any vertex $v_{k}, 1 \leq k \leq i$ and the vertex $v_{n+1}$. By applying the definition of the Gutman index to this pair of vertices we have the term:

$d_{J_{n+1}^{*}(x)}\left(v_{k}\right) d_{J_{n+1}^{*}(x)}\left(v_{n+1}\right) d_{J_{n+1}^{*}(x)}\left(v_{k}, v_{n+1}\right)=d_{J_{n}^{*}(x)}\left(v_{k}\right)(n-i)\left(d_{J_{n}^{*}(x)}\left(v_{k}, v_{n}\right)+1\right)$.

By applying this step $\forall v_{k}, 1 \leq k \leq i$ we obtain:

$$
\begin{aligned}
\sum_{k=1}^{i} d_{J_{n}^{*}(x)}\left(v_{k}\right)(n-i)\left(d_{J_{n}^{*}(x)}\right. & \left.\left(v_{k}, v_{n}\right)+1\right) \\
& =(n-i) \sum_{k=1}^{i} d_{J_{n}^{*}(x)}\left(v_{k}\right) d_{J_{n}^{*}(x)}\left(v_{k}, v_{n}\right)+i(n-i) .
\end{aligned}
$$

Step 5: Consider any vertex $v_{t}, i+1 \leq t \leq n$ and the vertex $v_{n+1}$. By applying the definition of the Gutman index to this pair of vertices we have the term:

$$
d_{J_{n+1}^{*}(x)}\left(v_{t}\right) d_{J_{n+1}^{*}(x)}\left(v_{n+1}\right) d_{J_{n+1}^{*}(x)}\left(v_{t}, v_{n+1}\right)=d_{J_{n}^{*}(x)}\left(v_{t}\right)(n-i) d_{J_{n}^{*}(x)}\left(v_{t}, v_{n}\right) .
$$


By applying this step $\forall v_{t}, i+1 \leq t \leq n$ we obtain:

$$
\sum_{t=i+1}^{n} d_{J_{n}^{*}(x)}\left(v_{t}\right)(n-i)=(n-i) \sum_{t=i+1}^{n} d_{J_{n}^{*}(x)}\left(v_{t}\right) .
$$

Final Summation Step: Adding Steps 1 to 5 and noting that:

$$
\begin{aligned}
\operatorname{Gut}\left(J_{n}^{*}(x)\right)= & \sum_{k=1}^{i-1} \sum_{q=k+1}^{i} d_{J_{n}^{*}(x)}\left(v_{k}\right) d_{J_{n}^{*}(x)}\left(v_{q}\right) d_{J_{n}^{*}(x)}\left(v_{k}, v_{q}\right) \\
& +\sum_{k=1}^{i} \sum_{t=i+1}^{n} d_{J_{n}^{*}(x)}\left(v_{k}\right) d_{J_{n}^{*}(x)}\left(v_{t}\right) d_{J_{n}^{*}(x)}\left(v_{k}, v_{t}\right) \\
& +\sum_{t=i+1}^{n-1} \sum_{q=t+1}^{n} d_{J_{n}^{*}(x)}\left(v_{t}\right) d_{J_{n}^{*}(x)}\left(v_{q}\right),
\end{aligned}
$$

provides the result:

$$
\begin{aligned}
& \operatorname{Gut}\left(J_{n+1}^{*}(x)\right)=\operatorname{Gut}\left(J_{n}^{*}(x)\right)+\sum_{k=1}^{i} \sum_{t=i+1}^{n} d_{J_{n}^{*}(x)}\left(v_{k}\right) d_{J_{n}^{*}(x)}\left(v_{k}, v_{t}\right) \\
& +\sum_{t=i+1}^{n-1} \sum_{q=t+1}^{n}\left(d_{J_{n}^{*}(x)}\left(v_{t}\right)+d_{J_{n}^{*}(x)}\left(v_{q}\right)\right)+(n-i)\left(\sum_{k=1}^{i} d_{J_{n}^{*}(x)}\left(v_{k}\right) d_{J_{n}^{*}(x)}\left(v_{k}, v_{n}\right)\right. \\
& \left.+\sum_{t=i+1}^{n} d_{J_{n}^{*}(x)}\left(v_{t}\right)\right)+(n-i-1)+i(n-i) .
\end{aligned}
$$

\section{The Gutman Index of the Edge-Joint between $J_{n}^{*}(x), n \in \mathbb{N}$ and $J_{m}^{*}(x), m \in \mathbb{N}$}

The concept of an edge-joint between two simple undirected graphs $G$ and $H$ is defined below.

Definition 3.1. The edge-joint of two simple undirected graphs $G$ and $H$ is the graph obtained by linking the edge $v u, v \in V(G), u \in V(H)$ and denoted, $G \rightsquigarrow v u H$. 
Note. $G \rightsquigarrow v u H=G \cup H+v u, v \in V(G), u \in V(H)$.

The next theorem provides $G u t\left(J_{n}^{*}(x) \rightsquigarrow v_{1} u_{1} J_{m}^{*}(x)\right)$ in terms of $G u t\left(J_{n}^{*}(x)\right)$ and $\operatorname{Gut}\left(J_{m}^{*}(x)\right)$. The edge-joint $J_{n}^{*}(x) \rightsquigarrow v_{1} u_{1} J_{m}^{*}(x)$ is called trivial. Edgejoints $J_{n}^{*}(x) \rightsquigarrow v_{i} u_{j} J_{m}^{*}(x), i \neq 1$ or $j \neq 1$ are called non-trivial. For families (classes) of graphs such as paths $P_{n}$, cycles $C_{n}$, complete graphs $K_{n}$, Jaco graphs $J_{n}(f(x))$, etc, the notation is abbreviated as $P_{n} \rightsquigarrow v u P_{m}=P_{n, m}^{\rightsquigarrow v u}$ and $J_{n}^{*}(f(x)) \rightsquigarrow v_{i} u_{j} J_{m}^{*}(f(x))=J_{n, m}^{\rightsquigarrow v_{i} u_{j}}$, etc.

Theorem 3.1. For the underlying graphs $J_{n}^{*}(x)$ and $J_{m}^{*}(x)$ of the finitie Jaco Graphs $J_{n}(x), J_{m}(x), n, m \in \mathbb{N}$ and $n \geq m \geq 2$ :

$$
\begin{gathered}
\operatorname{Gut}\left(J_{n}^{*}(x) \rightsquigarrow v_{1} u_{1} J_{m}^{*}(x)\right)=\operatorname{Gut}\left(J_{n, m_{1} u_{1}}^{\rightsquigarrow u_{1}}\right)=\operatorname{Gut}\left(J_{n}^{*}(x)\right)+\operatorname{Gut}\left(J_{m}^{*}(x)\right) \\
+\sum_{\ell=2}^{n} d_{J_{n}^{*}(x)}\left(v_{\ell}\right) d_{J_{n}^{*}(x)}\left(v_{1}, v_{\ell}\right)+\sum_{s=2}^{m} d_{J_{m}^{*}(x)}\left(u_{s}\right) d_{J_{m}^{*}(x)}\left(u_{1}, u_{s}\right) \\
\quad+\sum_{t=2}^{m}\left(d_{J_{n}^{*}(x)}\left(v_{1}\right)+1\right) d_{J_{m}^{*}(x)}\left(u_{t}\right)\left(d_{J_{m}^{*}(x)}\left(u_{1}, u_{t}\right)+1\right) \\
+\sum_{k=2}^{n} \sum_{t=2}^{m} d_{J_{n}^{*}(x)}\left(v_{k}\right) d_{J_{m}^{*}(x)}\left(u_{t}\right)\left(d_{J_{n}^{*}(x)}\left(v_{1}, v_{k}\right)+d_{J_{m}^{*}(x)}\left(u_{1}, u_{t}\right)+1\right)+4 .
\end{gathered}
$$

Proof. Consider the underlying Jaco graphs, $J_{n}^{*}(x), J_{m}^{*}(x)$, with $n, m \in \mathbb{N}$ and $n \geq m \geq 2$ with $J_{m}(x)$ having prime Jaconian vertex $u_{i}$. Also label the vertices of $J_{n}^{*}(x)$ and $J_{m}^{*}(x) ; v_{1}, v_{2}, v_{3}, \ldots, v_{n}$ and $u_{1}, u_{2}, u_{3}, \ldots, u_{m}$, respectively. Consider $J_{n, m}^{\rightsquigarrow v_{1} u_{1}}=J_{n}^{*}(x) \cup J_{m}^{*}(x)+v_{1} u_{1}$. Without loss of generality apply the piecewise definition:

$$
\begin{aligned}
& \operatorname{Gut}\left(J_{n, m}^{\rightsquigarrow v_{1} u_{1}}\right)=\sum_{k=1}^{n-1} \sum_{\ell=k+1}^{n} d_{J_{n, m}^{\rightsquigarrow v_{1} u_{1}}}\left(v_{k}\right) d_{J_{n, m}^{\rightsquigarrow v_{1} u_{1}}}\left(v_{\ell}\right) d_{J_{n, m}^{\rightsquigarrow v_{1} u_{1}}}\left(v_{k}, v_{\ell}\right) \\
& +\sum_{t=1}^{m-1} \sum_{s=t+1}^{m} d_{J_{n, m}^{\rightsquigarrow v_{1} u_{1}}}\left(u_{t}\right) d_{J_{n, m}^{\rightsquigarrow v_{1} u_{1}}}\left(u_{s}\right) d_{J_{n, m}^{\rightsquigarrow v_{1} u_{1}}}\left(u_{t}, u_{s}\right) \\
& +\sum_{k=1}^{n} \sum_{t=2}^{m} d_{J_{n, m}^{\curvearrowleft v_{1} u_{1}}}\left(v_{k}\right) d_{J_{n, m}^{\rightsquigarrow v_{1} u_{1}}}\left(u_{t}\right) d_{J_{n, m}^{\rightsquigarrow v_{1} u_{1}}}\left(v_{k}, u_{t}\right)+d_{J_{n, m}^{\curvearrowleft v_{1} u_{1}}}\left(v_{1}\right) \\
& d_{J_{n, m}^{\rightsquigarrow v_{1} u_{1}}}\left(u_{1}\right) d_{J_{n, m}^{\rightsquigarrow v_{1} u_{1}}}\left(v_{1}, u_{1}\right) .
\end{aligned}
$$

Step 1(a): Consider vertex $v_{1}$ and vertex $v_{\ell}, 2 \leq \ell \leq n$. By applying the definition of the Gutman index to this pair of vertices we have the term: 


$$
\begin{array}{r}
d_{J_{n, m}^{\rightsquigarrow v_{1} u_{1}}}\left(v_{1}\right) d_{J_{n, m}^{\rightsquigarrow v_{1} u_{1}}}\left(v_{\ell}\right) d_{J_{n, m}^{\rightsquigarrow v_{1} u_{1}}}\left(v_{1}, v_{\ell}\right)=\left(d_{J_{n}^{*}(x)}\left(v_{1}\right)+1\right) d_{J_{n}^{*}(x)}\left(v_{\ell}\right) d_{J_{n}^{*}(x)}\left(v_{1}, v_{\ell}\right) \\
=d_{J_{n}^{*}(x)}\left(v_{1}\right) d_{J_{n}^{*}(x)}\left(v_{\ell}\right) d_{J_{n}^{*}(x)}\left(v_{1}, v_{\ell}\right)+d_{J_{n}^{*}(x)}\left(v_{\ell}\right) d_{J_{n}^{*}(x)}\left(v_{1}, v_{\ell}\right)
\end{array}
$$

By applying this step $\forall v_{\ell}, 2 \leq \ell \leq n$ we obtain:

$$
\sum_{\ell=2}^{n} d_{J_{n}^{*}(x)}\left(v_{1}\right) d_{J_{n}^{*}(x)}\left(v_{\ell}\right) d_{J_{n}^{*}(x)}\left(v_{1}, v_{\ell}\right)+\sum_{\ell=2}^{n} d_{J_{n}^{*}(x)}\left(v_{\ell}\right) d_{J_{n}^{*}(x)}\left(v_{1}, v_{\ell}\right) .
$$

Step 1(b): For all ordered pairs of vertices $\left(v_{k}, v_{\ell}\right)_{k<\ell}$ with $2 \leq k \leq n-1$ and $3 \leq \ell \leq n$ we have that:

$$
\begin{aligned}
\sum_{k=2}^{n-1} \sum_{\ell=k+1}^{n} d_{J_{n, m}^{\rightsquigarrow v_{1} u_{1}}}\left(v_{k}\right) d_{J_{n, m}^{\rightsquigarrow v_{1} u_{1}}}\left(v_{\ell}\right) d_{J_{n, m}^{\rightsquigarrow v_{1} u_{1}}}\left(v_{k}, v_{\ell}\right) \\
=\sum_{k=2}^{n-1} \sum_{\ell=k+1}^{n} d_{J_{n}^{*}(x)}\left(v_{k}\right) d_{J_{n}^{*}(x)}\left(v_{\ell}\right) d_{J_{n}^{*}(x)}\left(v_{k}, v_{\ell}\right) .
\end{aligned}
$$

By applying this step $\forall\left(v_{k}, v_{\ell}\right)_{k<\ell}, 1 \leq k \leq n-1$ and $2 \leq \ell \leq n$, we obtain:

$$
\begin{array}{r}
\sum_{k=1}^{n-1} \sum_{\ell=k+1}^{n} d_{J_{n}^{*}(x)}\left(v_{k}\right) d_{J_{n}^{*}(x)}\left(v_{\ell}\right) d_{J_{n}^{*}(x)}\left(v_{k}, v_{\ell}\right)+\sum_{\ell=2}^{n} d_{J_{n}^{*}(x)}\left(v_{\ell}\right) d_{J_{n}^{*}(x)}\left(v_{1}, v_{\ell}\right) \\
=\operatorname{Gut}\left(J_{n}^{*}(x)\right)+\sum_{\ell=2}^{n} d_{J_{n}^{*}(x)}\left(v_{\ell}\right) d_{J_{n}^{*}(x)}\left(v_{1}, v_{\ell}\right) .
\end{array}
$$

Step 2: Similar to Step 1 we have that:

$$
\begin{aligned}
& \sum_{t=1}^{m-1} \sum_{s=t+1}^{m} d_{J_{n, m}^{\rightsquigarrow v_{1} u_{1}}}\left(u_{t}\right) d_{J_{n, m}^{\rightsquigarrow v_{1} u_{1}}}\left(u_{s}\right) d_{J_{n, m}^{\rightsquigarrow v_{1} u_{1}}}\left(u_{t}, u_{s}\right) \\
& =\sum_{t=1}^{m-1} \sum_{s=t+1}^{m} d_{J_{m}^{*}(x)}\left(u_{t}\right) d_{J_{m}^{*}(x)}\left(u_{s}\right) d_{J_{m}^{*}(x)}\left(u_{t}, u_{s}\right)+\sum_{s=2}^{m} d_{J_{m}^{*}(x)}\left(u_{s}\right) d_{J_{m}^{*}(x)}\left(u_{1}, u_{s}\right) \\
& =\operatorname{Gut}\left(J_{m}^{*}(x)\right)+\sum_{s=2}^{m} d_{J_{m}^{*}(x)}\left(u_{s}\right) d_{J_{m}^{*}(x)}\left(u_{1}, u_{s}\right) .
\end{aligned}
$$

Step 3: To conclude this step we will provide the next partial summation as a piecewise summation, to be: 


$$
\begin{aligned}
& \sum_{k=1}^{n} \sum_{t=2}^{m} d_{J_{n, m}^{\rightsquigarrow v_{1} u_{1}}}\left(v_{k}\right) d_{J_{n, m}^{\rightsquigarrow v_{1} u_{1}}}\left(u_{t}\right) d_{J_{n, m}^{\rightsquigarrow v_{1} u_{1}}}\left(v_{k}, u_{t}\right) \\
& =\sum_{t=2}^{m} d_{J_{n, m}^{\rightsquigarrow v_{1} u_{1}}}\left(v_{1}\right) d_{J_{n, m}^{\rightsquigarrow v_{1} u_{1}}}\left(u_{t}\right) d_{J_{n, m}^{\rightsquigarrow, v_{1} u_{1}}}\left(v_{1}, u_{t}\right) \\
& +\sum_{k=2}^{n} \sum_{t=2}^{m} d_{J_{n, m}^{\rightsquigarrow v_{1} u_{1}}}\left(v_{k}\right) d_{J_{n, m}^{\curvearrowleft, v_{1} u_{1}}}\left(u_{t}\right) d_{J_{n, m}^{\rightsquigarrow v_{1} u_{1}}}\left(v_{k}, u_{t}\right) .
\end{aligned}
$$

Step 3(a): Consider vertex $v_{1}$ and vertex $u_{t}, 2 \leq t \leq m$. By applying the definition of the Gutman index to this pair of vertices we have the term:

$$
\begin{aligned}
& d_{J_{n, m}^{\rightsquigarrow v_{1} u_{1}}}\left(v_{1}\right) d_{J_{n, m}^{\rightsquigarrow v_{1} u_{1}}}\left(u_{t}\right) d_{J_{n, m}^{\rightsquigarrow v_{1} u_{1}}}\left(v_{1}, u_{t}\right) \\
& \quad=\left(d_{J_{n}^{*}(x)}\left(v_{1}\right)+1\right) d_{J_{m}^{*}(x)}\left(u_{t}\right)\left(d_{J_{m}^{*}(x)}\left(u_{1}, u_{t}\right)+1\right) .
\end{aligned}
$$

By applying this step $\forall u_{t}, 2 \leq t \leq m$ we obtain:

$$
\sum_{t=2}^{m}\left(d_{J_{n}^{*}(x)}\left(v_{1}\right)+1\right) d_{J_{m}^{*}(x)}\left(u_{t}\right)\left(d_{J_{m}^{*}(x)}\left(u_{1}, u_{t}\right)+1\right)
$$

Step 3(b): Consider vertex $v_{k}, 2 \leq k \leq n$ and vertex $u_{t}, 2 \leq t \leq m$. By applying the definition of the Gutman index to this pair of vertices we have the term:

$$
\begin{aligned}
& d_{J_{n, m}^{\rightsquigarrow v_{1} u_{1}}}\left(v_{k}\right) d_{J_{n, m}^{\rightsquigarrow v_{1} u_{1}}}\left(u_{t}\right) d_{J_{n, m}^{\rightsquigarrow v_{1} u_{1}}}\left(v_{k}, u_{t}\right) \\
& \quad=d_{J_{n}^{*}(x)}\left(v_{k}\right) d_{J_{m}^{*}(x)}\left(u_{t}\right)\left(d_{J_{n}^{*}(x)}\left(v_{1}, v_{k}\right)+d_{J_{m}^{*}(x)}\left(u_{1}, u_{t}\right)+1\right) .
\end{aligned}
$$

By applying the step $\forall v_{k}, 2 \leq k \leq n$ and $\forall u_{t}, 2 \leq t \leq m$, we obtain:

$$
\sum_{k+2}^{n} \sum_{t=2}^{m} d_{J_{n}^{*}(x)}\left(v_{k}\right) d_{J_{m}^{*}(x)}\left(u_{t}\right)\left(d_{J_{n}^{*}(x)}\left(v_{1}, v_{k}\right)+d_{J_{m}^{*}(x)}\left(u_{1}, u_{t}\right)+1\right) .
$$

Step 4: It is easy to see that:

$$
d_{J_{n, m}^{\rightsquigarrow v_{1} u_{1}}}\left(v_{1}\right) d_{J_{n, m}^{\rightsquigarrow v_{1} u_{1}}}\left(u_{1}\right) d_{J_{n, m}^{\rightsquigarrow v_{1} u_{1}}}\left(v_{1}, u_{1}\right)=4 .
$$

Final Summation Step: Adding Steps 1 to 4 provides the result:

$$
G u t\left(J_{n, m}^{\rightsquigarrow v_{1} u_{1}}\right)=\operatorname{Gut}\left(J_{n}^{*}(x)\right)+G u t\left(J_{m}^{*}(x)\right)+\sum_{\ell=2}^{n} d_{J_{n}^{*}(x)}\left(v_{\ell}\right) d_{J_{n}^{*}(x)}\left(v_{1}, v_{\ell}\right)
$$




$$
\begin{gathered}
+\sum_{s=2}^{m} d_{J_{m}^{*}(x)}\left(u_{s}\right) d_{J_{m}^{*}(x)}\left(u_{1}, u_{s}\right)+\sum_{t=2}^{m}\left(d_{J_{n}^{*}(x)}\left(v_{1}\right)+1\right) d_{J_{m}^{*}(x)}\left(u_{t}\right)\left(d_{J_{m}^{*}(x)}\left(u_{1}, u_{t}\right)+1\right) \\
+\sum_{k=2}^{n} \sum_{t=2}^{m} d_{J_{n}^{*}(x)}\left(v_{k}\right) d_{J_{m}^{*}(x)}\left(u_{t}\right)\left(d_{J_{n}^{*}(x)}\left(v_{1}, v_{k}\right)+d_{J_{m}^{*}(x)}\left(u_{1}, u_{t}\right)+1\right)+4
\end{gathered}
$$

\section{Conclusion}

For the simple case $f(x)=x$ the calculation of the Gutman index for Jaco graph and the edge-joint between them is immediately complicated. Finding a result similar to Theorem 3.1 for $J_{n}^{*}(x) \rightsquigarrow v_{i} u_{j} J_{m}^{*}(x), i \neq 1$ or $j \neq 1$ (nontrivial edge-joints) remains open. The single most important challege is to find a closed formula for the number of edges in $J_{n}(x)$. Such closed formula will enable finding a closed formula for distances between given vertices and a simplied formula for many invariants of Jaco graphs might result from such finding. Hence, important open questions remain such as: Is there a closed formula for the number of edges of $J_{n}(x), n \in \mathbb{N}$ ? Is there a closed formula for the cardinality of the Jaconian set $\mathbb{J}\left(J_{n}(x)\right)$ of $J_{n}(x), n \in \mathbb{N}$ ? Is there a closed formula for $d_{J_{n}^{*}(x)}\left(v_{1}, v_{n}\right)$ in $J_{n}^{*}(x), n \in \mathbb{N}$ ?. Refer to [7] for further reading.

\section{References}

[1] V. Andova, D. Dimitrov, J. Fink, R. Škrekovski, Bounds on Gutman Index, MATCH Communications in Mathematical and in Computer Chemistry, Vol 67 (2012), pp 515524.

[2] J.A. Bondy, U.S.R. Murty,Graph Theory with Applications, Macmillan Press, London, (1976).

[3] P. Dankelmann, I. Gutman, S. Mukwembi, H.C. Swart, The edge-Wiener index of a graph, Discrete Mathematics, Vol 309 (2009), pp 3452-3457.

[4] I. Gutman, Selected properties of the Schultz molecular topological index, Journal of Chemical Information and Computer Sciences, Vol 34, (1994) pp 1087-1089.

[5] J. Kok, P. Fisher, B. Wilkens, M. Mabula, V. Mukungunugwa, Characteristics of Finite Jaco Graphs, $J_{n}(1), n \in \mathbb{N}$, arXiv: 1404.0484v1 [math.CO], 2 April 2014.

[6] J. Kok, P. Fisher, B. Wilkens, M. Mabula, V. Mukungunugwa, Characteristics of Jaco Graphs, $J_{\infty}(a), a \in \mathbb{N}$, arXiv: 1404.1714v1 [math.CO], 7 April 2014.

[7] J. Kok, C. Susanth, S.J. Kalayathankal, A Study on Linear Jaco Graphs, Journal of Informatics and Mathematical Sciences, Vol 7(2), (2015), 69-80. 
